\title{
Two-Sided First Exit Problem for Jump Diffusion Processes Having Jumps with a Mixture of Erlang Distribution*
}

\author{
Yuzhen Wen", Chuancun Yin \\ School of Mathematical Sciences, Qufu Normal University, Qufu, China \\ Email: "wenyzhen@163.com, ccyin@mail.qfnu.edu.cn
}

Received May 29, 2013; revised June 29, 2013; accepted July 7, 2013

Copyright (C) 2013 Yuzhen Wen, Chuancun Yin. This is an open access article distributed under the Creative Commons Attribution License, which permits unrestricted use, distribution, and reproduction in any medium, provided the original work is properly cited.

\begin{abstract}
In this paper, we consider the two-sided first exit problem for jump diffusion processes having jumps with rational Laplace transforms. We investigate the probabilistic property of conditional memorylessness, and drive the joint distribution of the first exit time from an interval and the overshoot over the boundary at the exit time.
\end{abstract}

Keywords: First Exit Time; Two-Sided Jumps; Jump Diffusion Process; Overshoot

\section{Introduction}

Consider the following jump diffusion process

$$
X_{t}=u+c t+\sigma W_{t}+\sum_{k=1}^{N_{t}} Y_{k}, t \geq 0,
$$

where the constant $u$ is the starting point of $\left\{X_{t}\right\}_{t>0}$, $c$ and $\sigma$ represent the drift and the volatility of the diffusion part, respectively, $\left\{W_{t}\right\}_{t \geq 0}$ is a standard Brownian motion with $W_{0}=0,\left\{N_{t}\right\}_{t \geq 0}$ is a Poisson process with rate $\lambda$, and the jumps sizes $\left\{Y_{1}, Y_{2}, \cdots\right\}$ are assumed to be i.i.d. real valued random variables with common density $p(x)$. Moreover, it is assumed that the random processes $\left\{W_{t}\right\}_{t \geq 0}, \quad\left\{N_{t}\right\}_{t \geq 0}$ and random variables $\left\{Y_{1}, Y_{2}, \cdots\right\}$ are mutually independent. In this paper we are interested in the density $p$ of following type

$$
\begin{aligned}
p(x)= & \sum_{j=1}^{J} \sum_{i=1}^{m_{j}} p_{i j} \frac{\rho_{j}^{i} x^{i-1}}{(i-1) !} \mathrm{e}^{-\rho_{j} x} I_{\{x>0\}} \\
& +\sum_{j=1}^{j} \sum_{i=1}^{\hat{m}_{j}} \hat{p}_{i j} \frac{\hat{\rho}_{j}^{i}|x|^{i-1}}{(i-1) !} \mathrm{e}^{\hat{\rho}_{j} x} I_{\{x<0\}},
\end{aligned}
$$

where $J, \hat{J}, m_{j}, \hat{m}_{j} \in \mathbb{N}, \quad p_{i j}, \hat{p}_{i j} \in \mathbb{R}^{+}, \quad \operatorname{Re}\left(\rho_{j}\right)>0$, $\operatorname{Re}\left(\hat{\rho}_{j}\right)>0$ and that $\rho_{i} \neq \rho_{j}, \quad \hat{\rho}_{i} \neq \hat{\rho}_{j}$ for all $i \neq j$. Moreover,

\footnotetext{
"This work was supported by the National Natural Science Foundation of China (No. 11171179) and Natural Science Foundation of Shandong Province (No. ZR2010AQ015).

${ }^{\#}$ Corresponding author.
}

$$
\sum_{j=1}^{J} \sum_{i=1}^{m_{j}} p_{i j}+\sum_{j=1}^{\hat{J}} \sum_{i=1}^{\hat{m}_{j}} \hat{p}_{i j}=1 .
$$

Define $\tau$ to be the first exit time of $X_{t}$ to two flat barriers $a$ and $b(a<b)$, i.e.

$$
\tau=\inf \left\{t \geq 0: X_{t} \geq b \text { or } X_{t} \leq a\right\} .
$$

Recently, one-sided and two-sided first exit problems for processes with two-sided jumps have attracted a lot of attentions in applied probability (see [1-7]). For example, Perry and Stadje [1] studied two-sided first exit time for processes with two-sided exponential jumps; Kou and Wang [2] studied the one-sided first passage times for a jump diffusion process with exponential positive and negative jumps. Cai [3] investigated the first passage time of a hyper-exponential jump diffusion process. Cai et al. [4] discussed the first passage time to two barriers of a hyper-exponential jump diffusion process. Closed form expressions are obtained in Kadankova and Veraverbeke [5] for the integral transforms of the joint distribution of the first exit time from an interval and the value of the overshoot through boundaries at the exit time for the Poisson process with an exponential component. For some related works, see Perry et al. [8], Cai and Kou [9], Lewis and Mordecki [10] and the references therein.

Motivated by works mentioned above, the main objective of this paper is to study the first exit time of the process (1.1) with jump density (1.2) from an interval 
and the overshoot over the boundary at the exit time. In Section 2, we study the roots of the generalized Lundberg equation and conditional memory lessness. The main results of this paper are given in Section 3.

\section{Preliminary Results}

It is easy to see that the infinitesimal generator of $\left\{X_{t}\right\}_{t \geq 0}$ is given by

$$
\begin{aligned}
(L \varphi)(x)= & \frac{1}{2} \sigma^{2} \varphi^{\prime \prime}(x)+c \varphi^{\prime}(x) \\
& +\lambda \int_{-\infty}^{\infty}[u(x+y)-u(x)] p(y) \mathrm{d} y
\end{aligned}
$$

for any twice continuously differentiable function $\varphi(x)$ and the Lévy exponent of $\left\{X_{t}\right\}_{t \geq 0}$ is given by

$$
\begin{aligned}
g(z)= & \frac{1}{t} \ln E \mathrm{e}^{z X}=\frac{1}{2} z^{2} \sigma^{2}+z c \\
& +\lambda\left(\sum_{j=1}^{J} \sum_{i=1}^{m_{j}} \frac{p_{i j}\left(\rho_{j}\right)^{i}}{\left(\rho_{j}-z\right)^{i}}+\sum_{j=1}^{j} \sum_{i=1}^{\hat{m}_{j}} \frac{\hat{p}_{i j}\left(\hat{\rho}_{j}\right)^{i}}{\left(\hat{\rho}_{j}+z\right)^{i}}-1\right) .
\end{aligned}
$$

By analytic continuation, the function $g(z)$ can be extended to the complex plane except at finitely many poles. In the following, we consider the resulting extension $G(z)$ of $g(z)$, i.e.,

$$
G(z)=\frac{1}{2} z^{2} \sigma^{2}+z C+\lambda\left(\sum_{j=1}^{J} \sum_{i=1}^{m_{j}} \frac{p_{i j}\left(\rho_{j}\right)^{i}}{\left(\rho_{j}-z\right)^{i}}+\sum_{j=1}^{j} \sum_{i=1}^{\hat{m}_{j}} \frac{\hat{p}_{i j}\left(\hat{\rho}_{j}\right)^{i}}{\left(\hat{\rho}_{j}+z\right)^{i}}-1\right), z \in C
$$

Let us denote $M=\sum_{j=1}^{J} m_{j}$ and $\hat{M}=\sum_{j=1}^{\hat{J}} \hat{m}_{j}$.

In [11], Kuznetsov has studied the roots of the equation $G(z)=\theta$. However, for this particular Lévy process $X$, we will give another simple proof for the roots of this equation.

Lemma 2.1. For fix $\theta>0$, the generalized CramérLundberg equation

$$
G(z)=\theta
$$

has $M+\hat{M}+2$ complex roots $\gamma_{1}(\theta), \gamma_{2}(\theta), \cdots, \gamma_{M+1}(\theta)$ with $\operatorname{Re}\left(\gamma_{i}(\theta)\right)>0$ for $i=1,2, \cdots, M+1 \quad$ and $\hat{\gamma}_{1}(\theta), \hat{\gamma}_{2}(\theta), \cdots, \hat{\gamma}_{\hat{M}+1}(\theta)$ with $\operatorname{Re}\left(\hat{\gamma}_{i}(\theta)\right)<0$ for $i=1,2, \cdots, \hat{M}+1$.

Proof. Let

$$
\begin{gathered}
G_{1}(z)=\frac{1}{2} z^{2} \sigma^{2}+z c-\lambda-\theta, z \in \mathbb{C}, \\
G_{2}(z)=\lambda\left(\sum_{j=1}^{J} \sum_{i=1}^{m_{j}} \frac{p_{i j}\left(\rho_{j}\right)^{i}}{\left(\rho_{j}-z\right)^{i}}+\sum_{j=1}^{j} \sum_{i=1}^{\hat{m}_{j}} \frac{\hat{p}_{i j}\left(\hat{\rho}_{j}\right)^{i}}{\left(\hat{\rho}_{j}+z\right)^{i}}\right), z \in \mathbb{C} .
\end{gathered}
$$

Firstly, we prove that for given $\theta>0, G(z)=\theta$ has $\hat{M}+1$ roots with negative real parts. Set $C_{r}^{-}=\left\{z:|z|=r, z \in C^{-}\right\}$with $r>\varepsilon+\max _{1 \leq j \leq \hat{J}}\left\{\left|\hat{\rho}_{j}\right|\right\}$,

where $\varepsilon$ is an arbitrary positive constant. Applying Rouchés theorem on the semi-circle $C_{r}^{-}$, consisting of the imaginary axis running from $-i r$ to ir and with radius $r$ running clockwise from ir to $-i r$. We let $r \rightarrow \infty$ and denote by $C^{-}$the limiting semi-circle. It is known that both $\left(\prod_{j=1}^{\hat{J}}\left(\hat{\rho}_{j}+z\right)^{\hat{m}_{j}}\right) G_{1}(z)$ and

$\left(\prod_{j=1}^{\hat{J}}\left(\hat{\rho}_{j}+z\right)^{\hat{m}_{j}}\right) G_{2}(z)$ are analytic in $C^{-}$. We want to show that
$\left|\left(\prod_{j=1}^{\hat{J}}\left(\hat{\rho}_{j}+z\right)^{\hat{m}_{j}}\right) G_{1}(z)\right|>\left|\left(\prod_{j=1}^{\hat{J}}\left(\hat{\rho}_{j}+z\right)^{\hat{m}_{j}}\right) G_{2}(z)\right|, z \in C^{-}$.

Notice that $\left|G_{1}(z)\right| \rightarrow \infty$ for $\operatorname{Re}(z) \rightarrow-\infty$, and $\left|G_{2}(z)\right| \leq \lambda \sum_{j=1}^{J} \sum_{i=1}^{m_{j}} \frac{\left|p_{i j}\right|\left|\rho_{j}\right|^{i}}{\varepsilon^{i}}+\lambda \sum_{j=1}^{\hat{J}} \sum_{i=1}^{\hat{m}_{j}} \frac{\left|\hat{p}_{i j}\right|\left|\hat{\rho}_{j}\right|^{i}}{\varepsilon^{i}}$ is bounded for $\operatorname{Re}(z) \rightarrow-\infty$. Hence, for $\operatorname{Re}(z) \rightarrow-\infty$,

$$
\left|\left(\prod_{j=1}^{\hat{J}}\left(\hat{\rho}_{j}+z\right)^{\hat{m}_{j}}\right) G_{1}(z)\right|>\left|\left(\prod_{j=1}^{\hat{J}}\left(\hat{\rho}_{j}+z\right)^{\hat{m}_{j}}\right) G_{2}(z)\right|
$$

on the boundary of the half circle in $C^{-}$. For $a \in R$, we have $\left|G_{2}(\mathrm{i} a)\right|<\lambda \quad$ (see Lewis and Mordecki [10]). On the other hand,

$$
\left|G_{1}(\mathrm{i} a)\right| \geq-\operatorname{Re} G_{1}(\mathrm{i} a)=\frac{1}{2} \sigma^{2} a^{2}+\lambda+\theta>\lambda .
$$

Thus we have $\left|G_{1}(\mathrm{i} a)\right|>\left|G_{2}(\mathrm{i} a)\right|$. Since $\left(\prod_{j=1}^{\hat{J}}\left(\hat{\rho}_{j}+z\right)^{\hat{m}_{j}}\right) G_{1}(z)$ has $\hat{M}+1$ roots with negative real parts, so equation $G(z)=\theta$ has $\hat{M}+1$ roots with negative real parts. Similarly, we can prove $G(z)=\theta, \theta>0$ has $M+1$ roots with positive real parts.

In the rest of this paper, we assume all the roots of equation $G(z)=\theta$ are distinct and denote

$\gamma_{i}(\theta)=\gamma_{i}(i=1,2, \cdots, M+1)$,

$\hat{\gamma}_{i}(\theta)=\hat{\gamma}_{i}(i=1,2, \cdots, \hat{M}+1)$ for notational simplicity, and denote $E^{u}$ (or $P^{u}$ in the sequel) representing the expectation (or probability) when $X_{t}$ starts from $u$. We denote a sequence of events

$$
K_{0}=\left\{\omega: X_{\tau}=b\right\}, G_{0}=\left\{\omega: X_{\tau}=a\right\}
$$

$K_{j i l}=\left\{\omega: X_{(\cdot)}\right.$ crosses $b$ at time $\tau$ by the $l$ th phase of $i$ th positive jump whose parameter is $\left.\rho_{j}\right\}$, 
$G_{j^{\prime} t^{\prime} \prime^{\prime}}=\left\{\omega: X_{(\cdot)}\right.$ crosses $a$ at time $\tau$ by the $l^{\prime}$ th phase of $i^{\prime}$ th negative jump whose parameter is $\left.\hat{\rho}_{j^{\prime}}\right\}$

for $j=1,2, \cdots, J, j^{\prime}=1,2, \cdots, \hat{J}, i=1, \cdots, m_{j}$, $i^{\prime}=1, \cdots, \hat{m}_{i^{\prime}}, l=1, \cdots, i$ and $l^{\prime}=1, \cdots, i^{\prime}$.

Theorem 2.2. For any $x>0$, we have

$$
\begin{gathered}
P^{u}\left(X_{\tau}-b \geq x \mid K_{j i l}\right)=\sum_{h=0}^{i-l} \frac{\left(\rho_{j} x\right)^{h}}{h !} \mathrm{e}^{-\rho_{j} x}, \\
P^{u}\left(X_{\tau}-a \leq-x \mid G_{j^{\prime} I^{\prime} I^{\prime}}\right)=\sum_{h=0}^{i^{\prime}-l^{\prime}} \frac{\left(\hat{\rho}_{j^{\prime}} x\right)^{h}}{h !} \mathrm{e}^{-\hat{\rho}_{j} x} .
\end{gathered}
$$

Furthermore, conditional on $\left\{\omega: \omega \in K_{j i l}\right\}$ $\left(\left\{\omega: \omega \in G_{j^{\prime} i^{\prime} l^{\prime}}\right\}\right)$, the stopping time $\tau$ is independent of the overshoot $X_{\tau}-b$ (the undershoot $X_{\tau}-a$ ). More precisely, for any $x>0$, we have

$$
\begin{aligned}
& P^{u}\left(\tau \leq t, X_{\tau}-b \geq x \mid K_{j i l}\right) \\
= & P^{u}\left(\tau \leq t, \mid K_{j i l}\right) \times P^{u}\left(X_{\tau}-b \geq x \mid K_{j i l}\right), \\
& P^{u}\left(\tau \leq t, X_{\tau}-a \leq-x \mid G_{j^{\prime} I^{\prime} l^{\prime}}\right) \\
= & P^{u}\left(\tau \leq t, \mid G_{j^{\prime} i^{\prime} l^{\prime}}\right) \times P^{u}\left(X_{\tau}-a \leq-x \mid G_{j^{\prime} i^{\prime} l^{\prime}}\right) .
\end{aligned}
$$

$$
\begin{aligned}
P_{n} & =P^{u}\left(T_{n}=\tau \leq t, X_{\tau}-b \geq x, I_{K_{j i l}}\right) \\
& =P^{u}\left(\max _{0 \leq s<T_{n}} X_{s}<b, X_{T_{n}}-b \geq x, T_{n}=\tau \leq t, I_{K_{j i l}}\right) \\
& \left.=E\left[P^{u}\left(X_{T_{n}}-b \geq x, I_{K_{j i l}} \mid \mathfrak{F}_{T_{n}-}, T_{n}\right) I_{\left(\max _{0 \leq s<T_{n}} X_{s}<b, T_{n}=\tau \leq t\right.}\right)\right] \\
& \left.=E\left[P^{u}\left(U_{u}\left(T_{n}\right)+Y_{1}+Y_{2}+\cdots+Y_{n}-b \geq x, I_{K_{j i l}} \mid \mathfrak{F}_{T_{n}-}, T_{n}\right) I_{\left(\max _{0 \leq s \leq T_{n}} X_{s}<b, T_{n}=\tau \leq t\right.}\right)\right] \\
& \left.=E\left[P^{u}\left(Y_{n} \geq b+x-\left(U_{u}\left(T_{n}\right)+\sum_{k=1}^{n-1} Y_{k}\right), I_{K_{j i l}} \mid \mathfrak{F}_{T_{n}-}, T_{n}\right) I_{\left(\max _{0 \leq s \leq T_{n}} X_{s}<b, T_{n}=\tau \leq t\right.}\right)\right] \\
& \left.=p_{i j} E\left[P^{u}\left(\sum_{k=l}^{i} V_{k} \geq b+x-U_{u}\left(T_{n}\right)-\sum_{k=1}^{n-1} Y_{k}-\sum_{k=1}^{l-1} V_{k}, V_{l} \geq b-U_{u}\left(T_{n}\right)-\sum_{k=1}^{n-1} Y_{j}-\sum_{k=1}^{l-1} V_{k} \mid \mathfrak{F}_{T_{n}-}, T_{n}\right) I_{\left(\max _{0 \leq s<T_{n}} X_{s}<b, T_{n}=\tau \leq t\right.}\right)\right] \\
& =\left(\sum_{h=0}^{i-l} \frac{\left(\rho_{j} x\right)^{h}}{h !} \mathrm{e}^{-\rho_{j} x}\right) P^{u}\left(T_{n}=\tau \leq t, I_{K_{j i l}}\right) .
\end{aligned}
$$

Thus we have

$$
\begin{aligned}
& P^{u}\left(\tau \leq t, X_{\tau}-b \geq x, I_{K_{j i l}}\right) \\
& =\left(\sum_{h=0}^{i-l} \frac{\left(\rho_{j} x\right)^{h}}{h !} \mathrm{e}^{-\rho_{j} x}\right) P^{u}\left(\tau \leq t, I_{K_{j i l}}\right) .
\end{aligned}
$$

Proof. Firstly, we prove (2.1) and (2.3). It suffices to show

$$
\begin{aligned}
& P^{u}\left(\tau \leq t, X_{\tau}-b \geq x, K_{j i l}\right) \\
& =\sum_{h=0}^{i-l} \frac{\left(\rho_{j} x\right)^{h}}{h !} \mathrm{e}^{-\rho_{j} x} P^{u}\left(\tau \leq t, K_{j i l}\right),
\end{aligned}
$$

since (2.1) can be obtained by letting $t \rightarrow \infty$ in (2.5) and then dividing both sides of the resulting equation by $P^{u}\left(K_{j i l}\right)$. It is known that an Erlang(n) random variable can be expressed as an independent sum of $n$ exponential random variables with same parameters. Let $V_{i},(i=1,2, \cdots, n)$ the $n$ independent exponentially distributed random variables with parameter $\rho_{i}$. Denote by $T_{1}, T_{2}, \cdots$ the arrival times of the Poisson process $N$, and let $\mathfrak{F}_{t-}=\sigma\left(\left\{X_{s}, 0 \leq s<t\right\}\right)$ be the field generated by process $X_{s}, 0 \leq s<t$. It follows that

$$
\begin{aligned}
& P^{u}\left(\tau \leq t, X_{\tau}-b \geq x, I_{K_{j i l}}\right) \\
& =\sum_{n=1}^{\infty} P^{u}\left(T_{n}=\tau \leq t, X_{\tau}-b \geq x, I_{K_{j i l}}\right)=\sum_{n=1}^{\infty} P_{n} .
\end{aligned}
$$

With $U_{u}(t)=u+c t+\sigma W(t)$, we have 


$$
\begin{gathered}
E^{u}\left[\mathrm{e}^{\alpha\left(X_{\tau}-b\right)} I\left(K_{j i l}\right)\right]=\left(\frac{\rho_{j}}{\rho_{j}-\alpha}\right)^{i+1-l}, \alpha \leq 0, \\
E^{u}\left[\mathrm{e}^{\alpha\left(X_{\tau}-a\right)} I\left(G_{j^{\prime} I^{\prime}}\right)\right]=\left(\frac{\hat{\rho}_{j}}{\hat{\rho}_{j}+\alpha}\right)^{i^{\prime}+1-l^{\prime}}, \alpha \geq 0 .
\end{gathered}
$$

Corollary 2.4. For any $x>0$, we have

$$
\begin{aligned}
& P^{u}\left(X_{\tau}-b \geq x \mid K_{j k}\right)=\sum_{h=0}^{k-1} \frac{\left(\rho_{j} x\right)^{h}}{h !} \mathrm{e}^{-\rho_{j} x}, \\
& P^{u}\left(X_{\tau}-a \leq-x \mid G_{j^{\prime} k^{\prime}}\right)=\sum_{h=0}^{k^{\prime}-1} \frac{\left(\hat{\rho}_{j^{\prime}} x\right)^{h}}{h !} \mathrm{e}^{-\hat{\rho}_{j} x}, \\
& P^{u}\left(\tau \leq t, X_{\tau}-b \geq x \mid K_{j k}\right) \\
& =P^{u}\left(\tau \leq t, \mid K_{j k}\right) \times P^{u}\left(X_{\tau}-b \geq x \mid K_{j k}\right), \\
& P^{u}\left(\tau \leq t, X_{\tau}-a \leq-x \mid G_{j^{\prime} k^{\prime}}\right) \\
& =P^{u}\left(\tau \leq t, \mid G_{j^{\prime} k^{\prime}}\right) \times P^{u}\left(X_{\tau}-a \leq-x \mid G_{j^{\prime} k^{\prime}}\right)
\end{aligned}
$$

where

$$
\begin{gathered}
f=\left(f(b), f_{11}^{u}, \cdots, f_{j k}^{u}, \cdots, f_{J m_{J}}^{u}, f(a), f_{11}^{d}, \cdots, f_{j^{\prime} k^{\prime}}^{d}, \cdots, f_{\hat{J}_{\hat{m}_{j}}}^{d}\right)^{\mathrm{T}}, \\
\pi=\left(E^{u}\left[\mathrm{e}^{-\theta \tau} I_{K_{0}}\right], \cdots, E^{u}\left[\mathrm{e}^{-\theta \tau} I_{K_{J m_{J}}}\right], E^{u}\left[\mathrm{e}^{-\theta \tau} I_{G_{0}}\right], \cdots, E^{u}\left[\mathrm{e}^{-\theta \tau} I_{G_{\hat{J}_{\hat{m}}}}\right]\right), \\
\varpi(u)=\left(\mathrm{e}^{\gamma_{1}(u-b)}, \mathrm{e}^{\gamma_{2}(u-b)}, \cdots, \mathrm{e}^{\gamma_{M+1}(u-b)}, \mathrm{e}^{\hat{\gamma}_{1}(u-a)}, \mathrm{e}^{\hat{\gamma}_{2}(u-a)}, \cdots, \mathrm{e}^{\hat{\gamma}_{\hat{M}+1}(u-a)}\right) .
\end{gathered}
$$

where

$$
\begin{gathered}
f_{j k}^{u}=\int_{0}^{\infty} f(y+b) \frac{\left(\rho_{j} y\right)^{k-1} \rho_{j} \mathrm{e}^{-\rho_{j} y}}{(k-1) !} \mathrm{d} y, j=1, \cdots, J, k=1, \cdots, m_{j}, \\
f_{j^{\prime} k^{\prime}}^{d}=\int_{-\infty}^{0} f(y+a) \frac{\left(-\hat{\rho}_{j} y\right)^{k^{\prime}-1} \hat{\rho}_{j} \mathrm{e}^{\hat{\rho}_{j} y}}{\left(k^{\prime}-1\right) !} \mathrm{d} y, j^{\prime}=1, \cdots, \hat{J}, k^{\prime}=1, \cdots, \hat{m}_{j^{\prime}} .
\end{gathered}
$$

Let

$$
\begin{aligned}
& C_{1}=\left(1,1, \cdots, 1, e^{\hat{\gamma}_{1}(b-a)}, e^{\hat{\gamma}_{2}(b-a)}, \cdots, e^{\hat{\gamma}_{M+1}(b-a)}\right), \quad \quad C_{2}=\left(\mathrm{e}^{\gamma_{1}(a-b)}, \mathrm{e}^{\gamma_{2}(a-b)}, \cdots, \mathrm{e}^{\gamma_{M+1}(a-b)}, 1,1, \cdots, 1\right), \\
& A_{i}=\left(\begin{array}{cccccc}
\frac{\rho_{i}}{\rho_{i}-\gamma_{1}} & \cdots & \frac{\rho_{i}}{\rho_{i}-\gamma_{M+1}} & \frac{\mathrm{e}^{\hat{\gamma}_{1}(b-a)} \rho_{i}}{\rho_{i}-\hat{\gamma}_{1}} & \cdots & \frac{\mathrm{e}^{\hat{r}_{\hat{M}+1}(b-a)} \rho_{i}}{\rho_{i}-\hat{\gamma}_{\hat{M}+1}} \\
\vdots & \ddots & \vdots & \vdots & \ddots & \vdots \\
\left(\frac{\rho_{i}}{\rho_{i}-\gamma_{1}}\right)^{m_{i}} & \cdots & \left(\frac{\rho_{i}}{\rho_{i}-\gamma_{M+1}}\right)^{m_{i}} & \frac{\mathrm{e}^{\hat{\gamma}_{1}(b-a)} \rho_{i}^{m_{i}}}{\left(\rho_{i}-\hat{\gamma}_{1}\right)^{m_{i}}} & \cdots & \frac{\mathrm{e}^{\hat{r}_{\hat{M}+1}(b-a)} \rho_{i}^{m_{i}}}{\left(\rho_{i}-\hat{\gamma}_{\hat{M}+1}\right)^{m_{i}}}
\end{array}\right), i=1,2, \cdots, J,
\end{aligned}
$$




$$
B_{i}=\left(\begin{array}{cccccc}
\frac{\mathrm{e}^{\gamma_{1}(a-b)} \hat{\rho}_{i}}{\hat{\rho}_{i}+\gamma_{1}} & \cdots & \frac{\mathrm{e}^{\gamma_{M+1}(a-b)} \hat{\rho}_{i}}{\hat{\rho}_{i}+\gamma_{M+1}} & \frac{\hat{\rho}_{i}}{\hat{\rho}_{i}+\hat{\gamma}_{1}} & \cdots & \frac{\hat{\rho}_{i}}{\hat{\rho}_{i}+\hat{\gamma}_{\hat{M}+1}} \\
\vdots & \ddots & \vdots & \vdots & \ddots & \vdots \\
\frac{\mathrm{e}^{\gamma_{1}(a-b)} \hat{\rho}_{i}^{\hat{m}_{i}}}{\left(\hat{\rho}_{i}+\gamma_{1}\right)^{\hat{m}_{i}}} & \cdots & \frac{\mathrm{e}^{\gamma_{M+1}(a-b)} \hat{\rho}_{i}^{\hat{m}_{i}}}{\left(\hat{\rho}_{i}+\gamma_{M+1}\right)^{\hat{m}_{i}}} & \left(\frac{\hat{\rho}_{i}}{\hat{\rho}_{i}+\hat{\gamma}_{1}}\right)^{\hat{m}_{i}} & \cdots & \left(\frac{\hat{\rho}_{i}}{\hat{\rho}_{i}+\hat{\gamma}_{\hat{M}+1}}\right)^{\hat{m}_{i}}
\end{array}\right), i=1,2, \cdots, \hat{J} .
$$

Define a matrix

$$
D=\left(\begin{array}{llllllll}
C_{1}^{T} & A_{1}^{T} & \cdots & A_{J}^{T} & C_{2}^{T} & B_{1}^{T} & \cdots & B_{\hat{J}}^{T}
\end{array}\right)^{T} .
$$

Theorem 3.1. Consider any nonnegative measurable function $f$ such that $f_{j k}^{u}<\infty$ and $f_{j^{\prime} k^{\prime}}^{d}<\infty$ for $j=1,2, \cdots, J, k=1, \cdots, m_{j}, \quad j^{\prime}=1, \cdots, \hat{J}, \quad k^{\prime}=1, \cdots, \hat{m}_{j^{\prime}}$. For any $\theta>0$ and $u \in(a, b)$, we have

$$
\begin{aligned}
E^{u}\left[\mathrm{e}^{-\theta \tau} f\left(X_{\tau}\right)\right]=\pi \mathbf{f}, & \text { Proof. By the law of total probabl } \\
E^{u}\left[\mathrm{e}^{-\theta \tau} f\left(X_{\tau}\right)\right]= & E^{u}\left[\mathrm{e}^{-\theta \tau} f\left(X_{\tau}\right) I_{K_{0}}\right]+\sum_{j=1}^{J} \sum_{i=1}^{m_{j}} \sum_{l=1}^{i} E^{u}\left[\mathrm{e}^{-\theta \tau} f\left(X_{\tau}\right) I_{K_{j i l}}\right] \\
& +E^{u}\left[\mathrm{e}^{-\theta \tau} f\left(X_{\tau}\right) I_{G_{0}}\right]+\sum_{j^{\prime}=1 i^{\prime}=1 l^{\prime}=1}^{j} \sum^{m_{j^{\prime}} i^{\prime}} E^{u}\left[\mathrm{e}^{-\theta \tau} f\left(X_{\tau}\right) I_{G_{j j^{\prime \prime}}}\right] \\
= & E^{u}\left[\mathrm{e}^{-\theta \tau} f\left(X_{\tau}\right) I_{K_{0}}\right]+\sum_{j=1}^{J} \sum_{k=1}^{m_{j}} E^{u}\left[\mathrm{e}^{-\theta \tau} f\left(X_{\tau}\right) I_{K_{j k}}\right] \\
& +E^{u}\left[\mathrm{e}^{-\theta \tau} f\left(X_{\tau}\right) I_{G_{0}}\right]+\sum_{j^{\prime}=1 k^{\prime}=1}^{j} \sum^{\hat{m}_{j^{\prime}}} E^{u}\left[\mathrm{e}^{-\theta \tau} f\left(X_{\tau}\right) I_{G_{j k^{\prime}}}\right] .
\end{aligned}
$$

where $\pi$ satisfies

$$
\pi D=\varpi .
$$

Moreover, when $D$ is a non-singular matrix, $\pi$ is the unique solution of (3.2), i.e.,

$$
\pi=\varpi \cdot D^{-1} .
$$

Proof. By the law of total probability, we have
It follows from Corollary 2.4, for $j=1,2, \cdots, J$, $k=1, \cdots, m_{j}, \quad j^{\prime}=1, \cdots, \hat{J}, \quad k^{\prime}=1, \cdots, \hat{m}_{j^{\prime}}$, we have

$$
\begin{gathered}
E^{u}\left[\mathrm{e}^{-\theta \tau} f\left(X_{\tau}\right) I_{K_{0}}\right]=E^{u}\left[\mathrm{e}^{-\theta \tau} I_{K_{0}}\right] f(b), \\
E^{u}\left[\mathrm{e}^{-\theta \tau} f\left(X_{\tau}\right) I_{G_{0}}\right]=E^{u}\left[\mathrm{e}^{-\theta \tau} I_{G_{0}}\right] f(a), \\
E^{u}\left[\mathrm{e}^{-\theta \tau} f\left(X_{\tau}\right) I_{K_{j k}}\right] \\
=E^{u}\left[\mathrm{e}^{-\theta \tau} I_{K_{j k}}\right] \int_{0}^{\infty} f(y+b) \frac{\left(\rho_{j} y\right)^{k-1} \rho_{j} \mathrm{e}^{-\rho_{j} y}}{(k-1) !} \mathrm{d} y, \\
E^{u}\left[\mathrm{e}^{-\theta \tau} f\left(X_{\tau}\right) I_{G_{j k^{\prime}}}\right] \\
=E^{u}\left[\mathrm{e}^{-\theta \tau} I_{G_{j^{\prime} k^{\prime}}}\right] \int_{-\infty}^{0} f(y+a) \frac{\left(-\hat{\rho}_{j} y\right)^{k^{\prime}-1} \hat{\rho}_{j} \mathrm{e}^{\hat{\rho}_{j} y}}{\left(k^{\prime}-1\right) !} \mathrm{d} y .
\end{gathered}
$$

Combining these equations, we get

$$
\begin{aligned}
& E^{u}\left[\mathrm{e}^{-\theta \tau} f\left(X_{\tau}\right)\right] \\
& =E^{u}\left[\mathrm{e}^{-\theta \tau} I_{K_{0}}\right] f(b)+\sum_{j=1}^{J} \sum_{k=1}^{m_{j}} E^{u}\left[\mathrm{e}^{-\theta \tau} I_{K_{j k}}\right] f_{j k}^{u} \\
& +E^{u}\left[\mathrm{e}^{-\theta \tau} I_{G_{0}}\right] f(a)+\sum_{j^{\prime}=1 k^{\prime}=1}^{\hat{m}} \sum^{\hat{m}_{j^{\prime}}} E^{u}\left[\mathrm{e}^{-\theta \tau} I_{G_{j^{\prime} k^{\prime}}}\right] f_{j^{\prime} k^{\prime}}^{d}
\end{aligned}
$$

The expressions for $E^{u}\left[\mathrm{e}^{-\theta \tau} I_{K_{0}}\right], E^{u}\left[\mathrm{e}^{-\theta \tau} I_{K_{j k}}\right]$, $E^{u}\left[\mathrm{e}^{-\theta \tau} I_{G_{0}}\right]$ and $E^{u}\left[\mathrm{e}^{-\theta \tau} I_{G_{j^{\prime k^{\prime}}}}\right]$ can be determined as follows. Let $\Delta$ denote the set of functions $g: R \rightarrow R$ such that $g(u)$ is twice continuously differentiable and bounded for $a<u<b$ with $g^{\prime}(u)$ and $g^{\prime \prime}(u)$ bounded for $a<u<b$. By applying Itô formula to the process $X_{t}$, we have that for $t \geq 0$ and $g \in \Delta$,

$$
g\left(X_{t \wedge \tau}\right)=g(u)+\int_{0}^{t \wedge \tau} L g\left(X_{s}\right) \mathrm{d} s+M_{t},
$$

where $M_{t}$ is a martingale with $M_{0}=0$. Note that we 
have $a<X_{s}<b$ as $s \leq \tau$.

For any $\theta>0$, we can easily obtain from the above equation that

$$
\begin{aligned}
& \mathrm{e}^{-\theta(t \wedge \tau)} g\left(X_{t \wedge \tau}\right) \\
& =g(u)+\int_{0}^{t \wedge \tau} \mathrm{e}^{-\theta s}\left(L g\left(X_{s}\right)-\theta g\left(X_{s}\right)\right) \mathrm{d} s+\int_{0}^{t \wedge \tau} \mathrm{e}^{-\theta s} \mathrm{~d} M_{t},
\end{aligned}
$$

where the last term of the above equation is a mean- 0 martingale. This implies that

$$
\begin{aligned}
& E^{u}\left[\mathrm{e}^{-\theta(t \wedge \tau)} g\left(X_{t \wedge \tau}\right)\right] \\
& =g(u)+E^{u}\left[\int_{0}^{t \wedge \tau} \mathrm{e}^{-\theta s}\left(L g\left(X_{s}\right)-\theta g\left(X_{s}\right)\right) \mathrm{d} s\right], t \geq 0 .
\end{aligned}
$$

By simple calculation, the function $g(x)=\mathrm{e}^{\beta x}$ with $G(\beta)=\theta$ and $\beta \in C$ satisfies $\operatorname{Lg}\left(X_{s}\right)-\theta g\left(X_{s}\right)=0$ for $a<x<b$. It follows from (3.4) that the process $\left\{\mathrm{e}^{-\theta(t \wedge \tau)+\beta X_{t \wedge \tau}}: t \geq 0\right\}$ is a martingale. Then

$$
\begin{aligned}
\mathrm{e}^{\beta u}= & E^{u}\left[\mathrm{e}^{-\theta \tau+\beta X_{\tau}}\right]=E^{u}\left[\mathrm{e}^{-\theta \tau} I_{K_{0}}\right] \mathrm{e}^{\beta b}+\sum_{j=1}^{J} \sum_{i=1}^{m_{j}} \sum_{l=1}^{i} E^{u}\left[\mathrm{e}^{-\theta \tau} I_{K_{j i l}}\right]\left(\frac{\rho_{j}}{\rho_{j}-\beta}\right)^{i+1-l} \mathrm{e}^{\beta b} \\
& +E^{u}\left[\mathrm{e}^{-\theta \tau} I_{G_{0}}\right] \mathrm{e}^{\beta a}+\sum_{j^{\prime}=1 i^{\prime}=1 l^{\prime}=1}^{\hat{J} \hat{m}_{j^{\prime}} i^{i^{\prime}}} E^{u}\left[\mathrm{e}^{-\hat{\rho} \tau} I_{G_{j^{\prime} t^{\prime}}}\right]\left(\frac{\hat{\rho}_{j^{\prime}}}{\hat{\rho}_{j^{\prime}}+\beta}\right)^{i^{\prime}+1-l^{\prime}} \mathrm{e}^{\beta a} \\
= & E^{u}\left[\mathrm{e}^{-\theta \tau} I_{K_{0}}\right] \mathrm{e}^{\beta b}+\sum_{j=1}^{J} \sum_{k=1}^{m_{j}} E^{u}\left[\mathrm{e}^{-\theta \tau} I_{K_{j k}}\right]\left(\frac{\rho_{j}}{\rho_{j}-\beta}\right)^{k} \mathrm{e}^{\beta b} \\
& +E^{u}\left[\mathrm{e}^{-\theta \tau} I_{G_{0}}\right] \mathrm{e}^{\beta a}+\sum_{j^{\prime}=1 k^{\prime}=1}^{j} \sum^{\hat{m}_{j^{\prime}}} E^{u}\left[\mathrm{e}^{-\hat{\rho} \tau} I_{G_{j^{\prime} k^{\prime}}}\right]\left(\frac{\hat{\rho}_{j^{\prime}}}{\hat{\rho}_{j^{\prime}}+\beta}\right)^{k^{\prime}} \mathrm{e}^{\beta a} .
\end{aligned}
$$

Setting $\beta=\gamma_{i}$ for $i=1,2, \cdots, M+1$ and $\beta=\hat{\gamma}_{i}$ for $i=1,2, \cdots, \hat{M}+1$ in (3.5), we have the following linear equations:

$$
\begin{aligned}
\mathrm{e}^{\gamma_{i} u}= & E^{u}\left[\mathrm{e}^{-\theta \tau+\gamma_{i} X_{\tau}}\right]=E^{u}\left[\mathrm{e}^{-\theta \tau} I_{K_{0}}\right] \mathrm{e}^{\gamma_{i} b}+\sum_{j=1}^{J} \sum_{k=1}^{m_{j}} E^{u}\left[\mathrm{e}^{-\theta \tau} I_{K_{j k}}\right]\left(\frac{\rho_{j}}{\rho_{j}-\gamma_{i}}\right)^{k} \mathrm{e}^{\gamma_{i} b} \\
& +E^{u}\left[\mathrm{e}^{-\theta \tau} I_{G_{0}}\right] \mathrm{e}^{\gamma_{i} a}+\sum_{j^{\prime}=1 k^{\prime}=1}^{j} \sum^{\hat{m}_{j^{\prime}}} E^{u}\left[\mathrm{e}^{-\theta \tau} I_{G_{j^{\prime}}}\right]\left(\frac{\hat{\rho}_{j^{\prime}}}{\hat{\rho}_{j^{\prime}}+\gamma_{i}}\right)^{k^{\prime}} \mathrm{e}^{\gamma_{i} a},
\end{aligned}
$$

and

$$
\begin{aligned}
\mathrm{e}^{\hat{\gamma}_{i} u}= & E^{u}\left[\mathrm{e}^{-\theta \tau \tau+\hat{\gamma}_{i} X_{\tau}}\right]=E^{u}\left[\mathrm{e}^{-\theta \tau} I_{K_{0}}\right] \mathrm{e}^{\hat{\gamma}_{i} b}+\sum_{j=1}^{J} \sum_{k=1}^{m_{j}} E^{u}\left[\mathrm{e}^{-\theta \tau} I_{K_{j k}}\right]\left(\frac{\rho_{j}}{\rho_{j}-\hat{\gamma}_{i}}\right)^{k} \mathrm{e}^{\hat{\gamma}_{i} b} \\
& +E^{u}\left[\mathrm{e}^{-\theta \tau} I_{G_{0}}\right] \mathrm{e}^{\hat{\gamma}_{i} a}+\sum_{j^{\prime}=1 k^{\prime}=1}^{\hat{m}} \sum^{\hat{m}_{j^{\prime}}} E^{u}\left[\mathrm{e}^{-\hat{\rho} \tau} I_{G_{j k^{\prime}}}\right]\left(\frac{\hat{\rho}_{j^{\prime}}}{\hat{\rho}_{j^{\prime}}+\hat{\gamma}_{i}}\right)^{k^{\prime}} \mathrm{e}^{\hat{\gamma}_{i} a} .
\end{aligned}
$$

Then the vector $\pi$ satisfies $\pi \cdot D=\varpi(u)$ and we have (3.1). If $D$ is non-singular, we have $\pi=\varpi(u) D^{-1}$. This completes the proof.

Corollary 3.2. For any

$\delta \in\left(-\min \left(\left|\hat{\rho}_{1}\right|,\left|\hat{\rho}_{2}\right|, \cdots,\left|\hat{\rho}_{J}\right|\right), \min \left(\left|\rho_{1}\right|,\left|\rho_{2}\right|, \cdots,\left|\rho_{\hat{\jmath}}\right|\right)\right)$, we have

$$
E^{u}\left[\mathrm{e}^{-\theta \tau+\delta X_{\tau}}\right]=e^{\delta b}\left(\sum_{i=1}^{M+1} \omega_{i} \mathrm{e}^{\gamma_{i}(u-b)}+\sum_{j=1}^{\hat{M}+1} v_{j} \hat{\gamma}^{\hat{\gamma}_{j}(u-a)}\right),
$$

where

$$
D\left(\omega_{1}, \omega_{2}, \cdots, \omega_{M+1}, v_{1}, v_{2}, \cdots, v_{\hat{M}+1}\right)^{\mathrm{T}}=J(\delta),
$$

and

$$
J(\delta)=\left(1,\left(\frac{\rho_{1}}{\rho_{1}-\delta}\right)^{m_{1}}, \cdots, \frac{\rho_{1}}{\rho_{1}-\delta}, \cdots,\left(\frac{\rho_{J}}{\rho_{J}-\delta}\right)^{m_{J}}, \cdots, \frac{\rho_{J}}{\rho_{J}-\delta}, \mathrm{e}^{\delta(a-b)},\left(\frac{\hat{\rho}_{1}}{\hat{\rho}_{1}+\delta}\right)^{\hat{m}_{1}} \mathrm{e}^{\delta(a-b)}, \cdots, \frac{\hat{\rho}_{\hat{\jmath}}}{\hat{\rho}_{\hat{J}}+\delta} \mathrm{e}^{\delta(a-b)}\right)^{\mathrm{T}} .
$$


Remark 3.3. When $m_{i}=1, n_{j}=1,(3.1)$ and (3.6) reduce to equation (6) and (15) of [4], respectively.

From Theorem 3.1, choosing $f\left(X_{\tau}\right)$ to be $I_{\left(X_{\tau}-b \geq 0\right)}$,
$I_{\left(X_{\tau}-a \leq 0\right)}, \quad I_{\left(X_{\tau}-b>y\right)}, \quad I_{\left(X_{\tau}-a<-y\right)}, \quad I_{\left(X_{\tau}=b\right)}, \quad I_{\left(X_{\tau}=a\right)}$ and $\mathrm{e}^{\delta X_{\tau}}$ respectively, we can obtain the following corollaries.

Corollary 3.4. 1) For any $\theta>0$, we have

$$
E^{u}\left[\mathrm{e}^{-\theta \tau} I_{\left(X_{\tau}-b \geq 0\right)}\right]=\left\{\begin{array}{lc}
0 & u \leq a \\
\sum_{i=1}^{M+1} \hat{\omega}_{i} \mathrm{e}^{-\gamma_{i}(b-u)}+\sum_{j=1}^{\hat{M}+1} \hat{v}_{j} \mathrm{e}^{-\hat{\gamma}_{j}(a-u)} & a<u<b, \\
1 & u \geq b
\end{array}\right.
$$

where

$$
\hat{\omega}^{\mathrm{T}}:=\left(\hat{\omega}_{1}, \hat{\omega}_{2}, \cdots, \hat{\omega}_{M+1}, \hat{v}_{1}, \hat{v}_{2}, \cdots, \hat{v}_{\hat{M}+1}\right)
$$

$$
I_{1}^{\mathrm{T}} \equiv(1,1, \cdots, 1,0,0, \cdots, 0)_{1 \times(M+\hat{M}+2)} .
$$

is determined by the linear system $D \hat{\omega}=I_{1}$. Here

2) For any $\theta>0$, we have

$$
E^{u}\left[\mathrm{e}^{-\theta \tau} I_{\left(X_{\tau}-a \leq 0\right)}\right]=\left\{\begin{array}{lc}
1 & u \leq a \\
\sum_{i=1}^{M+1} \hat{\hat{\omega}}_{i} \mathrm{e}^{-\gamma_{i}(b-u)}+\sum_{j=1}^{\hat{M}+1} \hat{\hat{v}}_{j} \mathrm{e}^{-\hat{\gamma}_{j}(a-u)} & b>u>a, \\
0 & u \geq b
\end{array}\right.
$$

where is determined by the linear system $D \hat{\hat{\omega}}=I_{2}$. Here

$$
\hat{\hat{\omega}}^{T}:=\left(\hat{\hat{\omega}}_{1}, \hat{\hat{\omega}}_{2}, \cdots, \hat{\hat{\omega}}_{M+1}, \hat{\hat{v}}_{1}, \hat{\hat{v}}_{2}, \cdots, \hat{\hat{v}}_{\hat{M}+1}\right)
$$

$$
I_{2}^{\mathrm{T}} \equiv(0,0, \cdots, 0,1,1, \cdots, 1)_{1 \times(M+\hat{M}+2)} .
$$

Corollary 3.5. 1) For $f\left(X_{\tau}\right)=I_{\left(X_{\tau}-a>y\right)}$ and any $\theta>0, y>0$, we have

$$
E^{u}\left[\mathrm{e}^{-\theta \tau} I_{\left(X_{\tau}-b>y\right)}\right]=\left\{\begin{array}{lc}
0 & u \in[b, b+y] \text { or } u \leq a \\
\sum_{i=1}^{M+1} \breve{\omega}_{i} \mathrm{e}^{-\gamma_{i}(b-u)}+\sum_{j=1}^{\hat{M}+1} \breve{v}_{j} \mathrm{e}^{-\hat{\gamma}_{j}(a-u)} & a<u<b \\
1 & u>b+y
\end{array},\right.
$$

where

$$
\breve{\omega}^{\mathrm{T}}=\left(\breve{\omega}_{1}, \breve{\omega}_{2}, \cdots, \breve{\omega}_{M+1}, \breve{v}_{1}, \breve{v}_{2}, \cdots, \breve{v}_{\hat{M}+1}\right)
$$

is determined by the linear system $D \breve{\omega}=\breve{I}_{1}$. Here

$$
\breve{I}_{1}^{\mathrm{T}} \equiv\left(0, \mathrm{e}^{-\rho_{1} y}, \cdots, \sum_{t=0}^{m_{1}-1} \frac{\left(\rho_{1} y\right)^{t}}{t !} \mathrm{e}^{-\rho_{1} y}, \cdots, \mathrm{e}^{-\rho_{J} y}, \cdots, \sum_{t=0}^{m_{J}-1} \frac{\left(\rho_{J} y\right)^{t}}{t !} \mathrm{e}^{-\rho_{J} y}, 0, \cdots, 0\right)_{1 \times(M+\hat{M}+2)} ;
$$

2) For $f\left(X_{\tau}\right)=I_{\left(X_{\tau}-a<-y\right)}$ and any $\theta>0, \quad y>0$, we have

$$
E^{u}\left[\mathrm{e}^{-\theta \tau} I_{\left(X_{\tau}-a<-y\right)}\right]=\left\{\begin{array}{lc}
1 & u<a-y \\
\sum_{i=1}^{M+1} \breve{\omega}_{i} \mathrm{e}^{-\gamma_{i}(b-u)}+\sum_{j=1}^{\hat{M}+1} \breve{V}_{j} \mathrm{e}^{-\hat{\gamma}_{j}(a-u)} & b>u>a \\
0 & u \in[a-y, a] \text { or } u \geq b
\end{array},\right.
$$

where

$$
\breve{\widetilde{\omega}}^{\mathrm{T}}=\left(\breve{\breve{\omega}}_{1}, \breve{\breve{\omega}}_{2}, \cdots, \breve{\widetilde{\omega}}_{M+1}, \breve{\breve{v}}_{1}, \breve{\bar{v}}_{2}, \cdots, \breve{\bar{v}}_{\hat{M}+1}\right)
$$

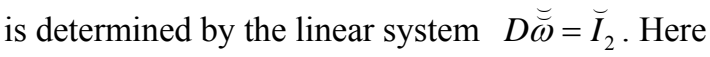




$$
\breve{I}_{2}^{\mathrm{T}} \equiv\left(0, \cdots, 0,0, \mathrm{e}^{-\hat{\rho}_{1} y}, \cdots, \sum_{t=0}^{\hat{m}_{1}-1} \frac{\left(\hat{\rho}_{1} y\right)^{t}}{t !} \mathrm{e}^{-\hat{\rho}_{1} y}, \cdots, \mathrm{e}^{-\hat{\rho}_{\hat{J}} y}, \cdots, \sum_{t=0}^{\hat{m}_{\hat{\jmath}}-1} \frac{\left(\hat{\rho}_{\hat{J}} y\right)^{t}}{t !} \mathrm{e}^{-\hat{\rho}_{\hat{J}} y}\right)_{1 \times(M+\hat{M}+2)} .
$$

Note that the difference of $E^{u}\left[\mathrm{e}^{-\theta \tau} I_{\left(X_{\tau}-b \geq 0\right)}\right]\left(E^{u}\left[\mathrm{e}^{-\theta \tau} I_{\left(X_{\tau}-a \leq 0\right)}\right]\right)$ and $E^{u}\left[\mathrm{e}^{-\theta \tau} I_{\left(X_{\tau}-b>0\right)}\right]\left(E^{u}\left[\mathrm{e}^{-\theta \tau} I_{\left(X_{\tau}-a<0\right)}\right]\right)$ is exactly $E^{u}\left[\mathrm{e}^{-\theta \tau} I_{\left(X_{\tau}=b\right)}\right]\left(E^{u}\left[\mathrm{e}^{-\theta \tau} I_{\left(X_{\tau}=a\right)}\right]\right)$. Thus we obtain the following results.

Corollary 3.6. 1) For $f\left(X_{\tau}\right)=I_{\left(X_{\tau}=b\right)}$, and for any $\theta>0$, we have

$$
E^{u}\left[\mathrm{e}^{-\theta \tau} I_{\left(X_{\tau}=b\right)}\right]=\left\{\begin{array}{lc}
0 & u>b \text { or } u \leq a \\
\sum_{i=1}^{M+1} \tilde{\omega}_{i} \mathrm{e}^{-\gamma_{i}(b-u)}+\sum_{j=1}^{\hat{M}+1} \tilde{v}_{j} \mathrm{e}^{-\hat{\gamma}_{j}(a-u)} & a<u<b \\
1 & u=b
\end{array},\right.
$$

where

$$
\tilde{\omega}^{\mathrm{T}}=\left(\tilde{\omega}_{1}, \tilde{\omega}_{2}, \cdots, \tilde{\omega}_{M+1}, \tilde{v}_{1}, \tilde{v}_{2}, \cdots, \tilde{v}_{\hat{M}+1}\right)
$$

is determined by the linear system $D \tilde{\omega}=\tilde{I}_{1}$. Here

$$
\tilde{I}_{1}^{\mathrm{T}} \equiv(1,0, \cdots, 0,0, \cdots, 0)_{1 \times(M+\hat{M}+2)} .
$$

2) For $f\left(X_{\tau}\right)=I_{\left(X_{\tau}=a\right)}$ and any $\theta>0, \quad y>0$, we have

$$
E^{u}\left[\mathrm{e}^{-\theta \tau} I_{\left(X_{\tau}=a\right)}\right]=\left\{\begin{array}{lc}
0 & u<a \text { or } u \geq b \\
\sum_{i=1}^{M+1} \bar{\omega}_{i} \mathrm{e}^{-\gamma_{i}(b-u)}+\sum_{j=1}^{\hat{M}+1} \bar{v}_{j} \mathrm{e}^{-\hat{\gamma}_{\hat{M}+1}(a-u)} & b>u>a, \\
1 & u=a
\end{array}\right.
$$

where

$$
\bar{v}^{\mathrm{T}}=\left(\bar{v}_{1}, \bar{v}_{2}, \cdots, \bar{v}_{M+1}, \bar{v}_{1}, \bar{v}_{2}, \cdots, \bar{v}_{\hat{M}+1}\right)
$$

is determined by the linear system $D \bar{v}=\bar{I}_{2}$. Here

$$
\bar{I}_{2}^{\mathrm{T}} \equiv(0,0, \cdots, 0,1,0, \cdots, 0)_{1 \times(M+\hat{M}+2)} .
$$

Then we have

To end the paper, we give an example.

$$
\begin{aligned}
& E^{u}\left[\mathrm{e}^{-\theta \tau} f\left(X_{\tau}\right)\right] \\
& =\omega_{1} \mathrm{e}^{\gamma_{1}(u-b)}+\omega_{2} \mathrm{e}^{\gamma_{2}(u-b)}+\omega_{3} \mathrm{e}^{\hat{1}_{1}(u-a)}+\omega_{4} \mathrm{e}^{\hat{\gamma}_{2}(u-a)},
\end{aligned}
$$

$p(x)=p_{11} \rho_{1} \mathrm{e}^{-\rho_{1} x} I_{(x>0)}+\hat{p}_{11} \hat{\rho}_{1} \mathrm{e}^{\hat{\rho}_{1} x} I_{(x<0)}$ and

$p_{11}+\hat{p}_{11}=1$, the equation $G(z)=\theta$ has 4 real roots:

where

$\gamma_{1}, \gamma_{2}, \hat{\gamma}_{1}$ and $\hat{\gamma}_{2}$

$\left(-\infty<\hat{\gamma}_{2}<-\hat{\rho}_{1}<\hat{\gamma}_{1}<0<\gamma_{1}<\rho_{1}<\gamma_{2}<\infty\right)$. Let

$$
D=\left(\begin{array}{cccc}
1 & 1 & \mathrm{e}^{\hat{\gamma}_{1}(b-a)} & \mathrm{e}^{\hat{\gamma}_{2}(b-a)} \\
\frac{\rho_{1}}{\rho_{1}-\gamma_{1}} & \frac{\rho_{1}}{\rho_{1}-\gamma_{2}} & \frac{\rho_{1} \mathrm{e}^{\hat{\gamma}_{1}(b-a)}}{\rho_{1}-\hat{\gamma}_{1}} & \frac{\rho_{1} \mathrm{e}^{\hat{\gamma}_{2}(b-a)}}{\rho_{1}-\hat{\gamma}_{2}} \\
\mathrm{e}^{\gamma_{1}(a-b)} & \mathrm{e}^{\gamma_{2}(a-b)} & 1 & 1 \\
\frac{\hat{\rho}_{1} \mathrm{e}^{\gamma_{1}(a-b)}}{\hat{\rho}_{1}+\gamma_{1}} & \frac{\hat{\rho}_{1} \mathrm{e}^{\gamma_{2}(a-b)}}{\hat{\rho}_{1}+\gamma_{2}} & \frac{\hat{\rho}_{1}}{\hat{\rho}_{1}+\hat{\gamma}_{1}} & \frac{\hat{\rho}_{1}}{\hat{\rho}_{1}+\hat{\gamma}_{1}}
\end{array}\right) .
$$

Denote $D^{-1}$ by

$$
\begin{aligned}
& \omega_{1}=\frac{d_{11} f(b)-d_{21} f_{11}^{u}+d_{31} f(a)-d_{41} f_{11}^{d}}{|D|}, \\
& \omega_{2}=\frac{-d_{12} f(b)+d_{22} f_{11}^{u}-d_{32} f(a)+d_{42} f_{11}^{d}}{|D|}, \\
& \omega_{3}=\frac{d_{13} f(b)-d_{23} f_{11}^{u}+d_{33} f(a)-d_{43} f_{11}^{d}}{|D|}, \\
& \omega_{4}=\frac{-d_{14} f(b)+d_{24} f_{11}^{u}-d_{34} f(a)+d_{44} f_{11}^{d}}{|D|},
\end{aligned}
$$




$$
\begin{aligned}
& f_{11}^{u}=\int_{0}^{\infty} f(y+b) \rho_{1} \mathrm{e}^{-\rho_{1} y} \mathrm{~d} y, \\
& f_{11}^{d}=\int_{-\infty}^{0} f(y+a) \hat{\rho}_{1} \mathrm{e}^{\hat{\rho}_{1} y} \mathrm{~d} y, \\
& |D|=\rho_{1} \hat{\rho}_{1} \frac{\left(\gamma_{2}-\gamma_{1}\right)\left(\hat{\gamma}_{1}-\hat{\gamma}_{2}\right)}{\left(\rho_{1}-\gamma_{1}\right)\left(\rho_{1}-\gamma_{2}\right)\left(\hat{\rho}_{1}+\hat{\gamma}_{1}\right)\left(\hat{\rho}_{1}+\hat{\gamma}_{2}\right)} \\
& +\left(\frac{\left(\gamma_{1}-\hat{\gamma}_{2}\right)\left(\hat{\gamma}_{1}-\gamma_{2}\right)}{\left(\hat{\rho}_{1}+\hat{\gamma}_{2}\right)\left(\rho_{1}-\hat{\gamma}_{1}\right)} \mathrm{e}^{\hat{\gamma}_{1}(b-a)}-\frac{\left(\gamma_{1}-\hat{\gamma}_{1}\right)\left(\gamma_{2}-\hat{\gamma}_{2}\right)}{\left(\hat{\rho}_{1}+\hat{\gamma}_{1}\right)\left(\rho_{1}-\hat{\gamma}_{2}\right)} \mathrm{e}^{\hat{\gamma}_{2}(b-a)}\right) \frac{\rho_{1} \hat{\rho}_{1} \mathrm{e}^{\gamma_{1}(a-b)}}{\left(\hat{\rho}_{1}+\gamma_{1}\right)\left(\rho_{1}-\gamma_{2}\right)} \\
& +\left(\frac{\left(\hat{\gamma}_{2}-\gamma_{2}\right)\left(\hat{\gamma}_{1}-\gamma_{1}\right)}{\left(\hat{\rho}_{1}+\hat{\gamma}_{2}\right)\left(\rho_{1}-\hat{\gamma}_{1}\right)} \mathrm{e}^{\hat{\gamma}_{1}(b-a)}-\frac{\left(\hat{\gamma}_{1}-\gamma_{2}\right)\left(\hat{\gamma}_{2}-\gamma_{1}\right)}{\left(\hat{\rho}_{1}+\hat{\gamma}_{1}\right)\left(\rho_{1}-\hat{\gamma}_{2}\right)} \mathrm{e}^{\hat{\gamma}_{2}(b-a)}\right) \frac{\rho_{1} \hat{\rho}_{1} \mathrm{e}^{\gamma_{2}(a-b)}}{\left(\hat{\rho}_{1}+\gamma_{2}\right)\left(\rho_{1}-\gamma_{1}\right)} \\
& +\frac{\left(\gamma_{1}-\gamma_{2}\right)\left(\hat{\gamma}_{2}-\hat{\gamma}_{1}\right)}{\left(\rho_{1}-\hat{\gamma}_{1}\right)\left(\rho_{1}-\hat{\gamma}_{2}\right)\left(\hat{\rho}_{1}+\gamma_{1}\right)\left(\hat{\rho}_{1}+\gamma_{2}\right)} \rho_{1} \hat{\rho}_{1} \mathrm{e}^{\left(\hat{\gamma}_{1}+\hat{\gamma}_{2}-\gamma_{1}-\gamma_{2}\right)(b-a)}, \\
& d_{11}=\frac{\rho_{1} \hat{\rho}_{1}\left(\hat{\gamma}_{1}-\hat{\gamma}_{2}\right)}{\left(\rho_{1}-\gamma_{2}\right)\left(\hat{\rho}_{1}+\hat{\gamma}_{1}\right)\left(\hat{\rho}_{1}+\hat{\gamma}_{2}\right)}+\left(\frac{\left(\gamma_{2}-\hat{\gamma}_{1}\right) \mathrm{e}^{\hat{\gamma}_{2}(b-a)}}{\left(\rho_{1}-\hat{\gamma}_{2}\right)\left(\hat{\rho}_{1}+\hat{\gamma}_{1}\right)}-\frac{\left(\gamma_{2}-\hat{\gamma}_{2}\right) \mathrm{e}^{\hat{\gamma}_{1}(b-a)}}{\left(\rho_{1}-\hat{\gamma}_{1}\right)\left(\hat{\rho}_{1}+\hat{\gamma}_{2}\right)}\right) \frac{\rho_{1} \hat{\rho}_{1} \mathrm{e}^{\gamma_{2}(a-b)}}{\hat{\rho}_{1}+\gamma_{2}}, \\
& d_{13}=\frac{\rho_{1} \hat{\rho}_{1}\left(\gamma_{2}-\hat{\gamma}_{2}\right) \mathrm{e}^{\gamma_{2}(a-b)}}{\left(\rho_{1}-\gamma_{1}\right)\left(\hat{\rho}_{1}+\gamma_{2}\right)\left(\hat{\rho}_{1}+\hat{\gamma}_{2}\right)}-\frac{\rho_{1} \hat{\rho}_{1}\left(\gamma_{1}-\hat{\gamma}_{2}\right) \mathrm{e}^{\gamma_{1}(a-b)}}{\left(\rho_{1}-\gamma_{2}\right)\left(\hat{\rho}_{1}+\gamma_{1}\right)\left(\hat{\rho}_{1}+\hat{\gamma}_{2}\right)}+\frac{\rho_{1} \hat{\rho}_{1}\left(\gamma_{1}-\gamma_{2}\right) \mathrm{e}^{\left(\gamma_{1}+\gamma_{2}-\hat{\gamma}_{2}\right)(a-b)}}{\left(\rho_{1}-\hat{\gamma}_{2}\right)\left(\hat{\rho}_{1}+\gamma_{1}\right)\left(\hat{\rho}_{1}+\gamma_{2}\right)}, \\
& d_{21}=\frac{\hat{\rho}_{1}\left(\hat{\gamma}_{1}-\hat{\gamma}_{2}\right)}{\left(\hat{\rho}_{1}+\hat{\gamma}_{1}\right)\left(\hat{\rho}_{1}+\hat{\gamma}_{2}\right)}+\left(\frac{\left(\gamma_{2}-\hat{\gamma}_{1}\right) \mathrm{e}^{\hat{\gamma}_{2}(b-a)}}{\hat{\rho}_{1}+\hat{\gamma}_{1}}-\frac{\left(\gamma_{2}-\hat{\gamma}_{2}\right) \mathrm{e}^{\hat{\gamma}_{1}(b-a)}}{\hat{\rho}_{1}+\hat{\gamma}_{2}}\right) \frac{\hat{\rho}_{1} \mathrm{e}^{\gamma_{2}(a-b)}}{\hat{\rho}_{1}+\gamma_{2}} \\
& d_{23}=\frac{\hat{\rho}_{1}\left(\hat{\gamma}_{2}-\gamma_{1}\right) \mathrm{e}^{\gamma_{1}(a-b)}}{\left(\hat{\rho}_{1}+\gamma_{1}\right)\left(\hat{\rho}_{1}+\hat{\gamma}_{2}\right)}+\frac{\hat{\rho}_{1}\left(\gamma_{2}-\hat{\gamma}_{2}\right) \mathrm{e}^{\gamma_{2}(a-b)}}{\left(\hat{\rho}_{1}+\gamma_{2}\right)\left(\hat{\rho}_{1}+\hat{\gamma}_{2}\right)}+\frac{\hat{\rho}_{1}\left(\gamma_{1}-\gamma_{2}\right) \mathrm{e}^{\left(\gamma_{1}+\gamma_{2}-\hat{\gamma}_{2}\right)(a-b)}}{\left(\hat{\rho}_{1}+\gamma_{1}\right)\left(\hat{\rho}_{1}+\gamma_{2}\right)}, \\
& d_{31}=\frac{\rho_{1} \hat{\rho}_{1}\left(\hat{\gamma}_{1}-\gamma_{2}\right) \mathrm{e}^{\hat{\gamma}_{1}(b-a)}}{\left(\rho_{1}-\gamma_{2}\right)\left(\rho_{1}-\hat{\gamma}_{1}\right)\left(\hat{\rho}_{1}+\hat{\gamma}_{2}\right)}-\frac{\rho_{1} \hat{\rho}_{1}\left(\hat{\gamma}_{2}-\gamma_{2}\right) \mathrm{e}^{\hat{\gamma}_{2}(b-a)}}{\left(\rho_{1}-\gamma_{2}\right)\left(\rho_{1}-\hat{\gamma}_{2}\right)\left(\hat{\rho}_{1}+\hat{\gamma}_{1}\right)}+\frac{\rho_{1} \hat{\rho}_{1}\left(\hat{\gamma}_{2}-\hat{\gamma}_{1}\right) \mathrm{e}^{\left(\hat{\gamma}_{1}+\hat{\gamma}_{2}-\gamma_{2}\right)(b-a)}}{\left(\rho_{1}-\hat{\gamma}_{1}\right)\left(\rho_{1}-\hat{\gamma}_{2}\right)\left(\hat{\rho}_{1}+\gamma_{2}\right)}, \\
& d_{33}=\frac{\rho_{1} \hat{\rho}_{1}\left(\gamma_{2}-\gamma_{1}\right)}{\left(\rho_{1}-\gamma_{1}\right)\left(\rho_{1}-\gamma_{2}\right)\left(\hat{\rho}_{1}+\hat{\gamma}_{2}\right)}+\left(\frac{\left(\gamma_{1}-\hat{\gamma}_{2}\right) \mathrm{e}^{\gamma_{2}(a-b)}}{\left(\rho_{1}-\gamma_{1}\right)\left(\hat{\rho}_{1}+\gamma_{2}\right)}-\frac{\left(\gamma_{2}-\hat{\gamma}_{2}\right) \mathrm{e}^{\gamma_{1}(a-b)}}{\left(\rho_{1}-\gamma_{2}\right)\left(\hat{\rho}_{1}+\gamma_{1}\right)}\right) \frac{\rho_{1} \hat{\rho}_{1} \mathrm{e}^{\hat{\gamma}_{2}(b-a)}}{\rho_{1}-\hat{\gamma}_{2}}, \\
& d_{41}=\frac{\rho_{1}\left(\hat{\gamma}_{1}-\gamma_{2}\right) \mathrm{e}^{\hat{\gamma}_{1}(b-a)}}{\left(\rho_{1}-\gamma_{2}\right)\left(\rho_{1}-\hat{\gamma}_{1}\right)}+\frac{\rho_{1}\left(\gamma_{2}-\hat{\gamma}_{2}\right) \mathrm{e}^{\hat{\gamma}_{2}(b-a)}}{\left(\rho_{1}-\gamma_{2}\right)\left(\rho_{1}-\hat{\gamma}_{2}\right)}+\frac{\rho_{1}\left(\hat{\gamma}_{2}-\hat{\gamma}_{1}\right) \mathrm{e}^{\left(\hat{\gamma}_{1}+\hat{\gamma}_{2}-\gamma_{2}\right)(b-a)}}{\left(\rho_{1}-\hat{\gamma}_{1}\right)\left(\rho_{1}-\hat{\gamma}_{2}\right)}, \\
& d_{43}=\frac{\rho_{1}\left(\gamma_{2}-\gamma_{1}\right)}{\left(\rho_{1}-\gamma_{1}\right)\left(\rho_{1}-\gamma_{2}\right)}+\left(\frac{\left(\gamma_{1}-\hat{\gamma}_{2}\right) \mathrm{e}^{\gamma_{2}(a-b)}}{\rho_{1}-\gamma_{1}}-\frac{\left(\gamma_{2}-\hat{\gamma}_{2}\right) \mathrm{e}^{\gamma_{1}(a-b)}}{\rho_{1}-\gamma_{2}}\right) \frac{\rho_{1} \mathrm{e}^{\hat{\gamma}_{2}(b-a)}}{\rho_{1}-\hat{\gamma}_{2}} .
\end{aligned}
$$

We define $d_{12}\left(d_{22}, d_{32}, d_{42}\right)$ and $d_{14}\left(d_{24}, d_{34}\right.$, $\left.d_{44}\right)$ as follows: let $d_{12}\left(d_{22}, d_{32}, d_{42}\right)$ be obtained from $d_{11}\left(d_{21}, d_{31}, d_{41}\right)$ by changing $\gamma_{2}$ to $\gamma_{1}$ in $d_{11}\left(d_{21} d_{31}, d_{41}\right)$; let $d_{14}\left(d_{24}, d_{34}, d_{44}\right)$ be obtained from $d_{13}\left(d_{23}, d_{33}, d_{43}\right)$ by changing $\hat{\gamma}_{2}$ to $\hat{\gamma}_{1}$ in $d_{13}\left(d_{23}, d_{33}, d_{43}\right)$.

- If $f\left(X_{\tau}\right)=\mathrm{e}^{\delta X_{\tau}}$, then we have

$$
\begin{aligned}
E^{u}\left[\mathrm{e}^{-\theta \tau+\delta X_{\tau}}\right]= & \mathrm{e}^{\delta b}\left(\omega_{1}^{\prime} \mathrm{e}^{\gamma_{1}(u-b)}+\omega_{2}^{\prime} \mathrm{e}^{\gamma_{2}(u-b)}\right. \\
& \left.+\omega_{3}^{\prime} \mathrm{e}^{\hat{\gamma}_{1}(u-a)}+\omega_{4}^{\prime} \mathrm{e}^{\hat{\gamma}_{2}(u-a)}\right), a<u<b,
\end{aligned}
$$

where

$$
\begin{gathered}
f(a)=\mathrm{e}^{\delta a}, f(b)=\mathrm{e}^{\delta b}, f_{11}^{u}=\mathrm{e}^{\delta b} \frac{\rho_{1}}{\rho_{1}-\delta}, f_{11}^{d}=\mathrm{e}^{\delta a} \frac{\hat{\rho}_{1}}{\hat{\rho}_{1}+\delta}, \\
\omega_{1}^{\prime}=\frac{d_{11}-d_{21} \frac{\rho_{1}}{\rho_{1}-\delta}+d_{31} \mathrm{e}^{\delta(a-b)}-d_{41} \mathrm{e}^{\delta(a-b)} \frac{\hat{\rho}_{1}}{\hat{\rho}_{1}+\delta}}{|D|} \\
\omega_{2}^{\prime}=\frac{-d_{12}+d_{22} \frac{\rho_{1}}{\rho_{1}-\delta}-d_{32} \mathrm{e}^{\delta(a-b)}+d_{42} \mathrm{e}^{\delta(a-b)} \frac{\hat{\rho}_{1}}{\hat{\rho}_{1}+\delta}}{|D|}
\end{gathered}
$$




$$
\begin{gathered}
\omega_{3}^{\prime}=\frac{d_{13}-d_{23} \frac{\rho_{1}}{\rho_{1}-\delta}+d_{33} e^{\delta(a-b)}-d_{43} e^{\delta(a-b)} \frac{\hat{\rho}_{1}}{\hat{\rho}_{1}+\delta}}{|D|} \\
\omega_{4}^{\prime}=\frac{-d_{14}+d_{24} \frac{\rho_{1}}{\rho_{1}-\delta}-d_{34} \mathrm{e}^{\delta(a-b)}+d_{44} \mathrm{e}^{\delta(a-b)} \frac{\hat{\rho}_{1}}{\hat{\rho}_{1}+\delta}}{|D|}
\end{gathered}
$$

- If $f\left(X_{\tau}\right)=I_{\left(X_{\tau}-b \geq 0\right)}$, then we have

$$
\begin{aligned}
E^{u}\left[\mathrm{e}^{-\theta \tau} I_{\left(X_{\tau}-b \geq 0\right)}\right]= & \hat{\omega}_{1} \mathrm{e}^{\gamma_{1}(u-b)}+\hat{\omega}_{2} \mathrm{e}^{\gamma_{2}(u-b)} \\
& +\hat{\omega}_{3} \mathrm{e}^{\hat{\gamma}_{1}(u-a)}+\hat{\omega}_{4} \mathrm{e}^{\hat{\gamma}_{2}(u-a)}, a<u<b,
\end{aligned}
$$

where

$$
\begin{gathered}
f(a)=0, f(b)=1, f_{11}^{u}=1, f_{11}^{d}=0, \\
\hat{\omega}_{1}=\frac{d_{11}-d_{21}}{|D|}, \hat{\omega}_{2}=\frac{-d_{12}+d_{22}}{|D|}, \\
\hat{\omega}_{3}=\frac{d_{13}-d_{23}}{|D|}, \hat{\omega}_{4}=\frac{-d_{14}+d_{24}}{|D|} ;
\end{gathered}
$$

- If $f\left(X_{\tau}\right)=I_{\left(X_{\tau}-a \leq 0\right)}$, then we have

$$
\begin{aligned}
E^{u}\left[\mathrm{e}^{-\theta \tau} I_{\left(X_{\tau}-a \leq 0\right)}\right]= & \hat{\hat{\omega}}_{1} \mathrm{e}^{\gamma_{1}(u-b)}+\hat{\hat{\omega}}_{2} \mathrm{e}^{\gamma_{2}(u-b)} \\
& +\hat{\hat{\omega}}_{3} \mathrm{e}^{\hat{\gamma}_{1}(u-a)}+\hat{\hat{\omega}}_{4} \mathrm{e}^{\hat{\gamma}_{2}(u-a)}, a<u<b,
\end{aligned}
$$

where

$$
\begin{gathered}
f(a)=1, f(b)=0, f_{11}^{u}=0, f_{11}^{d}=1, \\
\hat{\hat{\omega}}_{1}=\frac{d_{31}-d_{41}}{|D|}, \hat{\hat{\omega}}_{2}=\frac{-d_{32}+d_{42}}{|D|}, \\
\hat{\hat{\omega}}_{3}=\frac{d_{33}-d_{43}}{|D|}, \hat{\hat{\omega}}_{4}=\frac{-d_{34}+d_{44}}{|D|} ;
\end{gathered}
$$

- If $f\left(X_{\tau}\right)=I_{\left(X_{\tau}-b>y\right)}, y>0$, then we have

$$
\begin{aligned}
& E^{u}\left[\mathrm{e}^{-\theta \tau} I_{\left(X_{\tau}-b>y\right)}\right]= \breve{\omega}_{1} \mathrm{e}^{\gamma_{1}(u-b)}+\breve{\omega}_{2} \mathrm{e}^{\gamma_{2}(u-b)} \\
&+\breve{\omega}_{3} \overline{\mathrm{e}}^{\hat{\gamma}_{1}(u-a)}+\breve{\omega}_{4} \mathrm{e}^{\hat{\gamma}_{2}(u-a)}, a<u<b, \\
& f(a)=0, \quad f(b)=0, \quad f_{11}^{u}=\mathrm{e}^{-\rho_{1} y}, \quad f_{11}^{d}=0,
\end{aligned}
$$$$
\hat{\omega}_{1}=\frac{d_{11}-d_{21}}{|D|}=\frac{\frac{\rho_{1} \hat{\rho}_{1}\left(\hat{\gamma}_{1}-\hat{\gamma}_{2}\right)}{\left(\rho_{1}-\gamma_{2}\right)\left(\hat{\rho}_{1}+\hat{\gamma}_{1}\right)\left(\hat{\rho}_{1}+\hat{\gamma}_{2}\right)}-\frac{\hat{\rho}_{1}\left(\hat{\gamma}_{1}-\hat{\gamma}_{2}\right)}{\left(\hat{\rho}_{1}+\hat{\gamma}_{1}\right)\left(\hat{\rho}_{1}+\hat{\gamma}_{2}\right)}}{\rho_{1} \hat{\rho}_{1} \frac{\left(\gamma_{2}-\gamma_{1}\right)\left(\hat{\gamma}_{1}-\hat{\gamma}_{2}\right)}{\left(\rho_{1}-\gamma_{1}\right)\left(\rho_{1}-\gamma_{2}\right)\left(\hat{\rho}_{1}+\hat{\gamma}_{1}\right)\left(\hat{\rho}_{1}+\hat{\gamma}_{2}\right)}}=\frac{\gamma_{2}\left(\rho_{1}-\gamma_{1}\right)}{\rho_{1}\left(\gamma_{2}-\gamma_{1}\right)},
$$

$$
\begin{aligned}
& \breve{\omega}_{1}=\frac{-d_{21} \mathrm{e}^{-\rho_{1} y}}{|D|}, \breve{\omega}_{2}=\frac{d_{22} \mathrm{e}^{-\rho_{1} y}}{|D|}, \\
& \breve{\omega}_{3}=\frac{-d_{23} \mathrm{e}^{-\rho_{1} y}}{|D|}, \breve{\omega}_{4}=\frac{d_{24} \mathrm{e}^{-\rho_{1} y}}{|D|} ;
\end{aligned}
$$

- If $f\left(X_{\tau}\right)=I_{\left(X_{\tau}-a<-y\right)}, \quad y>0$, then we have

$$
\begin{aligned}
E^{u}\left[\mathrm{e}^{-\theta \tau} I_{\left(X_{\tau}-a<-y\right)}\right]= & \breve{\mathscr{\omega}}_{1} \mathrm{e}^{\gamma_{1}(u-b)}+\breve{\widetilde{\omega}}_{2} \mathrm{e}^{\gamma_{2}(u-b)} \\
& +\breve{\widetilde{\omega}}_{3} \mathrm{e}^{\hat{\gamma}_{1}(u-a)}+\breve{\widetilde{\omega}}_{4} \mathrm{e}^{\hat{\gamma}_{2}(u-a)}, a<u<b,
\end{aligned}
$$

where

$$
f(a)=0, \quad f(b)=0, f_{11}^{u}=0, \quad f_{11}^{d}=\mathrm{e}^{-\hat{\rho}_{1} y},
$$

$$
\begin{aligned}
& \omega_{1}=\frac{-d_{41} \mathrm{e}^{-\hat{\rho}_{1} y}}{|D|}, \omega_{2}=\frac{d_{42} \mathrm{e}^{-\hat{\rho}_{1} y}}{|D|}, \\
& \omega_{3}=\frac{-d_{43} \mathrm{e}^{-\hat{\rho}_{1} y}}{|D|}, \omega_{4}=\frac{d_{44} \mathrm{e}^{-\hat{\rho}_{1} y}}{|D|} ;
\end{aligned}
$$

- If $f\left(X_{\tau}\right)=I_{\left(X_{\tau}=b\right)}$, then we have

$$
\begin{aligned}
E^{u}\left[\mathrm{e}^{-\theta \tau} I_{\left(X_{\tau}=b\right)}\right]= & \tilde{\omega}_{1} \mathrm{e}^{\gamma_{1}(u-b)}+\tilde{\omega}_{2} \mathrm{e}^{\gamma_{2}(u-b)} \\
& +\tilde{\omega}_{3} \mathrm{e}^{\hat{\gamma}_{1}(u-a)}+\tilde{\omega}_{4} \mathrm{e}^{\hat{\gamma}_{2}(u-a)}, a<u<b,
\end{aligned}
$$

where

$$
f(a)=0, f(b)=1, f_{11}^{u}=0, f_{11}^{d}=0,
$$

$$
\tilde{\omega}_{1}=\frac{d_{11}}{|D|}, \quad \tilde{\omega}_{2}=\frac{-d_{12}}{|D|}, \quad \tilde{\omega}_{3}=\frac{d_{13}}{|D|}, \quad \tilde{\omega}_{4}=\frac{-d_{14}}{|D|} ;
$$

- If $f\left(X_{\tau}\right)=I_{\left(X_{\tau}=a\right)}$, then we have

$E^{u}\left[\mathrm{e}^{-\theta \tau} I_{\left(X_{\tau}=a\right)}\right]=\bar{\omega}_{1} \mathrm{e}^{\gamma_{1}(u-b)}+\bar{\omega}_{2} \mathrm{e}^{\gamma_{2}(u-b)}$

where

$$
+\bar{\omega}_{3} \mathrm{e}^{\hat{\gamma}_{1}(u-a)}+\bar{\omega}_{4} \mathrm{e}^{\hat{\gamma}_{2}(u-a)}, a<u<b,
$$

$$
\begin{gathered}
f(a)=1, f(b)=0, f_{11}^{u}=0, f_{11}^{d}=0, \\
\bar{\omega}_{1}=\frac{d_{31}}{|D|}, \bar{\omega}_{2}=\frac{-d_{32}}{|D|}, \bar{\omega}_{3}=\frac{d_{33}}{|D|}, \bar{\omega}_{4}=\frac{-d_{34}}{|D|} .
\end{gathered}
$$

When $a \rightarrow-\infty$, we have 


$$
\begin{aligned}
& \hat{\omega}_{2}=\frac{-d_{12}+d_{22}}{|D|}=\frac{\frac{\rho_{1} \hat{\rho}_{1}\left(\hat{\gamma}_{1}-\hat{\gamma}_{2}\right)}{\left(\rho_{1}-\gamma_{1}\right)\left(\hat{\rho}_{1}+\hat{\gamma}_{1}\right)\left(\hat{\rho}_{1}+\hat{\gamma}_{2}\right)}-\frac{\hat{\rho}_{1}\left(\hat{\gamma}_{1}-\hat{\gamma}_{2}\right)}{\left(\hat{\rho}_{1}+\hat{\gamma}_{1}\right)\left(\hat{\rho}_{1}+\hat{\gamma}_{2}\right)}}{\rho_{1} \hat{\rho}_{1} \frac{\left(\gamma_{2}-\gamma_{1}\right)\left(\hat{\gamma}_{1}-\hat{\gamma}_{2}\right)}{\left(\rho_{1}-\gamma_{1}\right)\left(\rho_{1}-\gamma_{2}\right)\left(\hat{\rho}_{1}+\hat{\gamma}_{1}\right)\left(\hat{\rho}_{1}+\hat{\gamma}_{2}\right)}}=-\frac{\gamma_{1}\left(\rho_{1}-\gamma_{2}\right)}{\rho_{1}\left(\gamma_{2}-\gamma_{1}\right)}, \\
& \breve{\omega}_{1}=\frac{-d_{21} \mathrm{e}^{-\rho_{1} y}}{|D|}=\frac{-\frac{\hat{\rho}_{1}\left(\hat{\gamma}_{1}-\hat{\gamma}_{2}\right)}{\left(\hat{\rho}_{1}+\hat{\gamma}_{1}\right)\left(\hat{\rho}_{1}+\hat{\gamma}_{2}\right)} \mathrm{e}^{-\rho_{1} y}}{\rho_{1} \hat{\rho}_{1} \frac{\left(\gamma_{2}-\gamma_{1}\right)\left(\hat{\gamma}_{1}-\hat{\gamma}_{2}\right)}{\left(\rho_{1}-\gamma_{1}\right)\left(\rho_{1}-\gamma_{2}\right)\left(\hat{\rho}_{1}+\hat{\gamma}_{1}\right)\left(\hat{\rho}_{1}+\hat{\gamma}_{2}\right)}}=-\frac{\left(\rho_{1}-\gamma_{1}\right)\left(\rho_{1}-\gamma_{2}\right)}{\rho_{1}\left(\gamma_{2}-\gamma_{1}\right)} \mathrm{e}^{-\rho_{1} y} \text {, } \\
& \breve{\omega}_{2}=\frac{d_{22} \mathrm{e}^{-\rho_{1} y}}{|D|}=\frac{\frac{\hat{\rho}_{1}\left(\hat{\gamma}_{1}-\hat{\gamma}_{2}\right)}{\left(\hat{\rho}_{1}+\hat{\gamma}_{1}\right)\left(\hat{\rho}_{1}+\hat{\gamma}_{2}\right)} \mathrm{e}^{-\rho_{1} y}}{\rho_{1} \hat{\rho}_{1} \frac{\left(\gamma_{2}-\gamma_{1}\right)\left(\hat{\gamma}_{1}-\hat{\gamma}_{2}\right)}{\left(\rho_{1}-\gamma_{1}\right)\left(\rho_{1}-\gamma_{2}\right)\left(\hat{\rho}_{1}+\hat{\gamma}_{1}\right)\left(\hat{\rho}_{1}+\hat{\gamma}_{2}\right)}}=\frac{\left(\rho_{1}-\gamma_{1}\right)\left(\rho_{1}-\gamma_{2}\right)}{\rho_{1}\left(\gamma_{2}-\gamma_{1}\right)} \mathrm{e}^{-\rho_{1} y},
\end{aligned}
$$

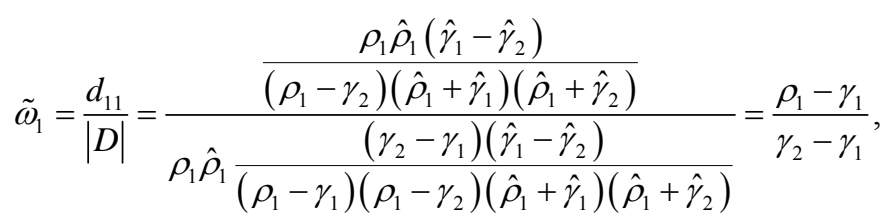

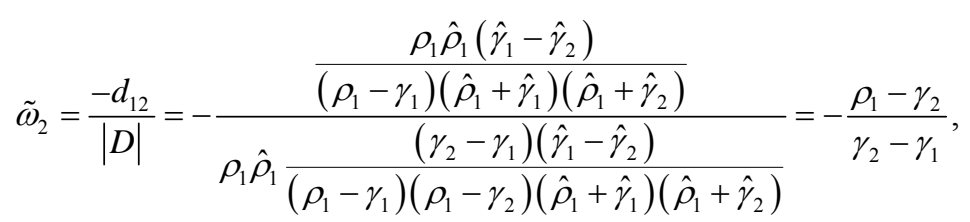$$
\hat{\omega}_{3}=\hat{\omega}_{4}=\breve{\omega}_{3}=\breve{\omega}_{4}=\tilde{\omega}_{3}=\tilde{\omega}_{4}=0 .
$$

Therefore, we have

$$
\begin{gathered}
E^{0}\left[\mathrm{e}^{-\theta \tau}\right]=\frac{\gamma_{2}\left(\rho_{1}-\gamma_{1}\right)}{\rho_{1}\left(\gamma_{2}-\gamma_{1}\right)} \mathrm{e}^{-\gamma_{1} b}-\frac{\gamma_{1}\left(\rho_{1}-\gamma_{2}\right)}{\rho_{1}\left(\gamma_{2}-\gamma_{1}\right)} \mathrm{e}^{-\gamma_{2} b}, \\
E^{0}\left[\mathrm{e}^{-\theta \tau} I_{\left(X_{\tau}-b>y\right)}\right] \\
=\frac{\left(\rho_{1}-\gamma_{1}\right)\left(\rho_{1}-\gamma_{2}\right)}{\rho_{1}\left(\gamma_{2}-\gamma_{1}\right)}\left(\mathrm{e}^{-\gamma_{2} b}-\mathrm{e}^{-\gamma_{1} b}\right) \mathrm{e}^{-\rho_{1} y}, \\
E^{0}\left[\mathrm{e}^{-\theta \tau} I_{\left(X_{\tau}=b\right)}\right]=\frac{\rho_{1}-\gamma_{1}}{\gamma_{2}-\gamma_{1}} \mathrm{e}^{-\gamma_{1} b}-\frac{\rho_{1}-\gamma_{2}}{\gamma_{2}-\gamma_{1}} \mathrm{e}^{-\gamma_{2} b} .
\end{gathered}
$$

These results are all consistent with that of Theorem 3.1 of Kou and Wang [2] for the one-sided exit problem of the doubly exponential jump diffusion process.

\section{REFERENCES}

[1] D. Perry and W. Stadje, "Risk Analysis for a Stochastic Cash Management Model with Two Types of Customers," Insurance: Mathematics and Economics, Vol. 26, No. 1, 2000, pp. 25-36. doi:10.1016/S0167-6687(99)00037-2

[2] S. G. Kou and H. Wang, "First Passage Times of a Jump
Diffusion Process," Advances in Applied Probability, Vol. 35, No. 2, 2003, pp. 504-531. doi:10.1239/aap/1051201658

[3] N. Cai, "On First Passage Times of a Hyper-Exponential Jump Diffusion Process," Operations Research Letters, Vol. 37, No. 2, 2009, pp. 127-134. doi:10.1016/j.orl.2009.01.002

[4] N. Cai, N. Chen and X. W. Wan, "Pricing Double-Barrier Options under a Flexible Jump Diffusion Model," Operations Research Letters, Vol. 37, No. 3, 2009, pp. 163-167. doi:10.1016/i.orl.2009.02.006

[5] T. Kadankova and N. Veraverbeke, "On Several TwoBondary Problems for a Particular Class of Lévy Processes," Journal of Theoretical Probability, Vol. 20, No. 4, 2007, pp. 1073-1085. doi:10.1007/s10959-007-0088-8

[6] S. Fourati, "Explicit Solutions of the Exit Problem for a Class of Lévy Processes; Applications to the Pricing of Double-Barrier Options," Stochastic Processes and their Applications, Vol. 122, No. 3, 2012, pp. 1034-1067. doi:10.1016/j.spa.2011.09.008

[7] M. Jacobsen, "The Time to Ruin for a Class of Markov Additive Risk Process with Two-Sided Jumps," Advances in Applied Probability, Vol. 37, No. 4, 2005, pp. 963-992. doi:10.1239/aap/1134587749

[8] D. Perry, W. Stadje and S. Zacks, "Contributions to the Theory of First-Exit Times of Some Compound Processes in Queueing Theory," Queueing Systems, Vol. 33, No. 4, 1999, pp. 369-379. doi:10.1023/A:1019140616021 
[9] N. Cai and S. G. Kou, "Option Pricing under a Mixed-Exponential Jump Diffusion Model," Management Science, Vol. 57, No. 11, 2011, pp. 2067-2081. doi:10.1287/mnsc. 1110.1393

[10] A. L. Lewis and E. Mordecki, "Wiener-Hopf Factorization for Lévy Processes Having Positive Jumps with Rational Transforms," Journal of Applied Probability, Vol.
45, No. 1, 2008, pp. $118-134$. doi:10.1239/jap/1208358956

[11] A. Kuznetsov, "On the Distribution of Exponential Functionals for Lévy Processes with Jumps of Rational Transform," Stochastic Processes and their Applications, Vol. 122, No. 2, 2012, pp. 654-663.

doi:10.1016/j.spa.2011.09.007 